\title{
Modelling Non-Gaussian Surfaces and Misalignment for Condition Monitoring of Journal Bearings
}

\author{
Jiaojiao Ma ${ }^{\mathrm{a}, \mathrm{b}}$, Chao $\mathrm{Fu}^{\mathrm{b}}$, Hao Zhang ${ }^{\mathrm{a}}$, Fulei $\mathrm{Chu}^{\mathrm{c}}$, Zhanqun Shia ${ }^{\mathrm{a}}$, Fengshou Gu ${ }^{\mathrm{b}}$, Andrew D. Ball ${ }^{\mathrm{b}}$

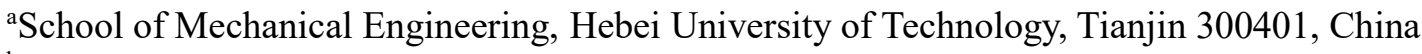 \\ ${ }^{b}$ Centre for Efficiency and Performance Engineering, University of Huddersfield, Queensgate, Huddersfield, \\ HD1 3DH, UK \\ ${ }^{\mathrm{c} D e p a r t m e n t ~ o f ~ P r e c i s i o n ~ I n s t r u m e n t s ~ a n d ~ M e c h a n o l o g y, ~ T s i n g h u a ~ U n i v e r s i t y, ~ B e i j i n g ~ 100084, ~ C h i n a ~}$
}

\begin{abstract}
In the hydrodynamic lubricated journal bearing system, the surface roughness and angular misalignment are two critical factors that affect bearing's performance. In this paper, the coupled effects of different non-Gaussian properties and misalignments are investigated on the performance of journal bearings. Christensen's stochastic model is extended by improving the probability density function of random roughness heights, which incorporates the Gram-Charlier expansion including skewness and kurtosis. In comparison with a Gaussian surface, the non-Gaussian rough surface has more significant influence on the bearing static performance. The negative skewness and large kurtosis increase the load capacity and decrease the friction coefficient. According to the simulations and experiments, non-Gaussian properties have more impact on the performance than misalignment when the journal bearing is operated in hydrodynamic lubrication regime based on different pressure distributions and vibration responses. These novel findings provide the basis for monitoring the conditions of hydrodynamic journal bearings.
\end{abstract}

Keywords: Journal bearing, non-Gaussian rough surface, misalignment, performance. 


\section{Introduction}

Hydrodynamic lubricated journal bearings are commonly used to undertake the heavy external load or high rotary speed in rotating machinery, such as pumps, engines, compressors, turbines, fans, etc. In some cases, the magnetic fluid is employed as the active lubricant to improve the performance of the relative sliding surfaces $[1,2]$. Lubricants present in the gap between the journal and bearing to reduce friction and noise. The journal inside bearing drives the lubricant and form fluid film to separate the relative sliding surfaces and balance the external loads [3-6]. It is ideal that the journal works in bearing with their centre lines parallel. In fact, the angular misalignment of journal is hardly ruled out for the oil film thickness in a micro-level. The misalignment can be caused by many factors, e.g., asymmetrical loading, elastic deformation of shaft, thermal distortion of shaft, manufacturing and installation errors and so on $[7,8]$. This leads to the decrease of minimum film thickness and even to asperity contact on the relative sliding surfaces and consequently increased frictional wear. Hence, it is critical to study the effects of misalignment on the performance of journal bearings.

Many researchers have analysed the journal misalignment influence on the static and dynamic characteristics of bearings. MaKee and McKee [9] firstly noticed that the noncentral loads lead to a change of the peak pressure in same direction based on experiment. Dubois et al. [10] investigated the misalignment leading to the peak pressure increase and concluded that the peak pressure was also related to the bearing clearance and length-to-diameter ratios. Pinkus and Bupara [11] derived the equation of film thickness considering the misaligned influence on a grooved journal bearing and compared the features of bearing, which were caused by the various misalignment in magnitude and direction. Buckholz and Lin [12] discussed the change of load-carrying capacity and the boundary condition of cavitation caused by misalignment in journal bearing lubricated by non-Newtonian fluid. Vijayaraghavan and Keith $[13,14]$ analysed the effects of thermohydrodynamic and then considering cavitation and starvation on a misaligned journal bearing. Sun and Gui [15] derived the expression of oil film thickness combined with the effect of journal misalignment, which was resulted from the journal deformation under external loads. Lv and Jiao [16] presented the misalignment in vertical direction and discussed the change of friction coefficient with varying rotating speeds including the effect of turbulent under the same external loads. In addition, many researchers have investigated that 
the angular misalignment of journal led to the deterioration of bearing performance [17-21].

Generally, the discussion of static or dynamic characteristics of a misaligned journal was completed based on the premise of journal and bearing owing to smooth surfaces. It is well known that the change of bearing performance is caused by factors that will result in the alteration of oil film thickness. The value of surface roughness in practice is on the same order with the oil film thickness. Therefore, the surface roughness should not be overlooked as one of critical parameters varying the actual gap between journal and bearing, particularly with a misaligned journal. Abdel-Latif and Mokhtar [22] concluded that the hydrodynamic performance of full finite journal bearing was affected by both journal misalignment and surface roughness. Guha [23] addressed the influence of isotropic surface roughness on the static performance of misaligned journal bearing based on the Christensen's model. Sharma et al. [24] studied the combined effect of rough surface and misalignment in the performance analysis of hole-entry hybrid journal bearings based on the average Reynolds equation. Sun and Deng et al. [25] found that the effects of oil viscosity-pressure relationship and surface roughness were obvious as the misaligned journal worked at a high eccentricity ratio and concluded the thermal influence remarkable with the large misalignment angle. Subsequently, they [26] added the effects of elastic deformations on the hydrodynamic performance of misaligned journal bearing. Recently, Zhu and Sun et al. [27] showed that the maximum pressure, load capacity and misalignment moment increase as the surface roughness and misalignment angle increase.

As mentioned above, the pattern of rough surface was assumed to follow the Gaussian distribution. Practically, the machined surfaces by different manufacturing processes, i.e., turning, grinding, drilling, shaping and electrolytic machining, which also included wear effects, exhibit a non-Gaussian rough surface and the distribution of surface roughness heights is stochastic [28-31]. To accurately describe it, skewness and kurtosis of surface roughness parameters should be considered. In literature, the nonGaussian surface generally was applied in the contact analysis [32-37]. In hydrodynamic lubrication regime, Li and Zhang et al. [38] extended the stochastic methods of Christensen to analyse the nonGaussian properties effects and investigated the static performance of slider bearing changes with skewness and kurtosis varied. Wang and Liu [39] revealed the fluid load capability of water lubricated tittle thrust pad bearing not only affected by the standard deviation of the surface roughness but also by skewness and kurtosis. Clearly, the non-Gaussian rough surface effects cannot be ignored in gaining 
a more realistic understanding of bearing's behaviours.

However, the research with respect to the combined influences of the non-Gaussian rough surface and misalignment on the bearing performance is not available until now. The present paper is devoted to investigating the performance of the misaligned journal bearing with various surfaces patterns according to the alteration of skewness and kurtosis. The oil film thickness is firstly introduced based on the journal misalignment and then the random heights of surface roughness is added. In addition, according to Christensen's stochastic methods [40], Reynolds equation is further extended by introducing the Gram-Charlier expression, which is utilized to approximate the Probability Density Function (PDF) of the random roughness heights including the skewness and kurtosis of the rough surface. Then, the static characteristics of misaligned journal bearings in terms of the maximum pressure, fluid load capacity, friction coefficient, end leakage flow-rate and misaligned moment are evaluated with the influence of non-Gaussian surfaces. Finally, the validation experiments are carried out to verify the effectiveness of the improved model.

\section{Modelling}

\subsection{Non-Gaussian and misalignment model of journal bearings}

The cylindrical plain journal bearing with the misalignment caused by the shaft deformation under external load $W$ is shown in Fig. 1(a). For the smooth surfaces, the approach in Ref. [13] is utilized to describe the oil film thickness considering the journal misalignment as follows

$$
h_{r}=c+e_{0} \cos \left(\Phi-\Phi_{0}\right)+\left(z-\frac{l}{2}\right) \tan \beta \cos \left(\Phi-\Phi_{0}-\alpha\right)
$$

where $c$ is the radial clearance between the journal and bearing. $e_{0}$ and $\Phi_{0}$ denote the eccentricity and attitude angle at the axial mid-plane of the bearing. $\alpha$ means the angle between the journal rear centreline projection and eccentricity vector. $z$ denotes the axial coordinate. $\beta$ is the misalignment angle, which can be calculated by $\beta=\arctan \left(e^{\prime} / l\right)$. Here, $e^{\prime}$ is the length of the projection of the misaligned centre-line on the end-plane of bearing. $l$ denotes the bearing width.

Considering the rough surfaces of the journal and bearing, the local oil film thickness is defined by

$$
h=h_{r}+\delta_{J}+\delta_{B}
$$

where $\delta_{J}$ and $\delta_{B}$ are the roughness amplitudes of journal and bearing surfaces as shown in Fig. 1(b). 
In order to simplify the calculation, asperity heights on the surfaces of journal and bearing are defined by a variable $\delta$. Hence, the nominal roughness height is $\delta=\delta_{J}+\delta_{B}$, and the standard deviation of the roughness height is $\sigma=\sqrt{\sigma_{J}^{2}+\sigma_{B}^{2}}$.

Submitting the dimensionless parameters

$$
\varepsilon_{0}=\frac{e_{0}}{c}, \quad \zeta=\frac{z}{c}-\frac{l}{2 c}, \quad H_{r}=\frac{h_{r}}{c}, \quad \Delta=\frac{\delta}{c}
$$

into Eq. (2), then the dimensionless oil film thickness can be obtained by

$$
H=H_{r}+\Delta=1+\varepsilon_{0} \cos \left(\Phi-\Phi_{0}\right)+\zeta \tan \beta \cos \left(\Phi-\Phi_{0}-\alpha\right)+\Delta
$$

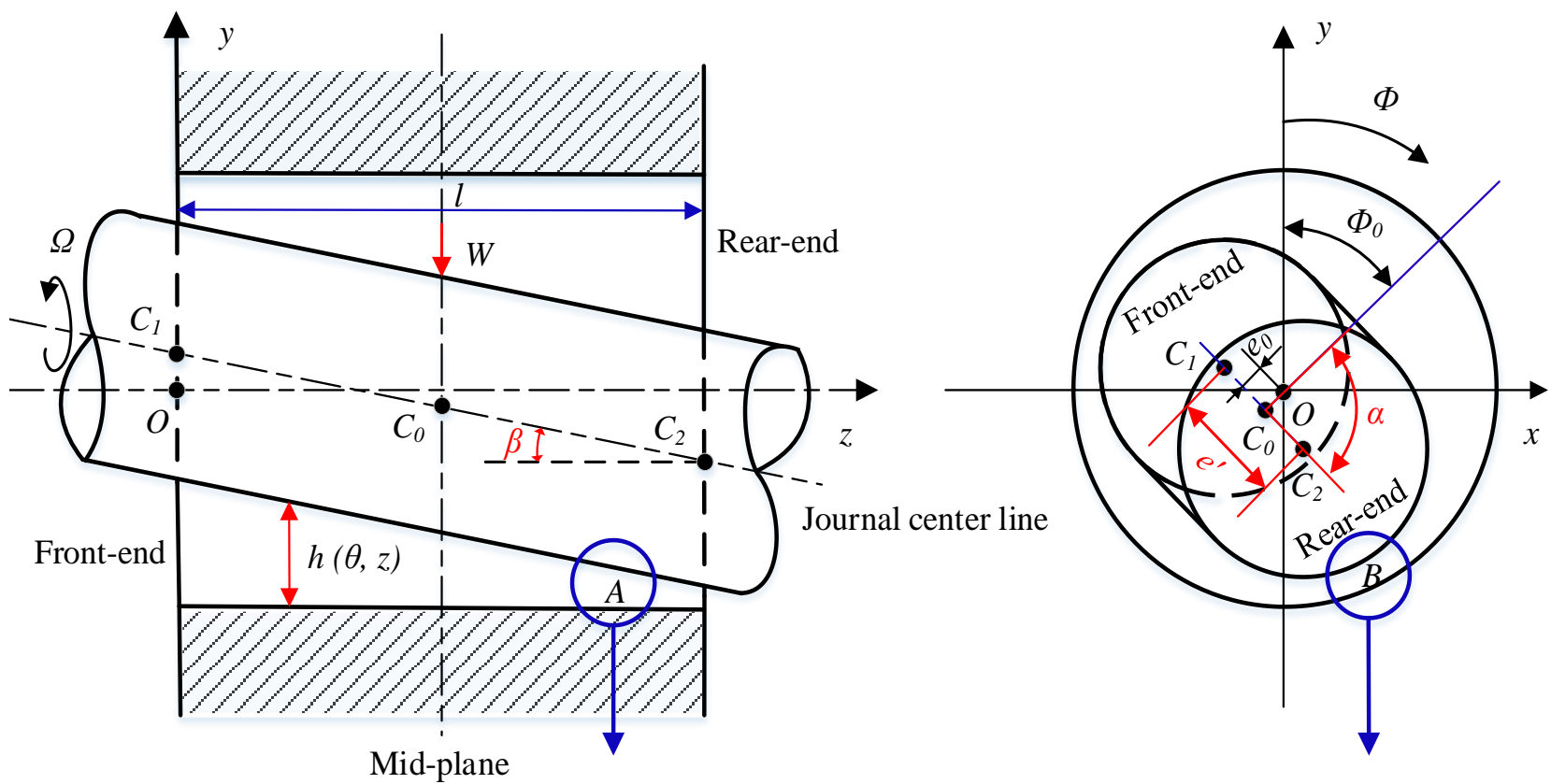

(a) A misaligned jounral bearing

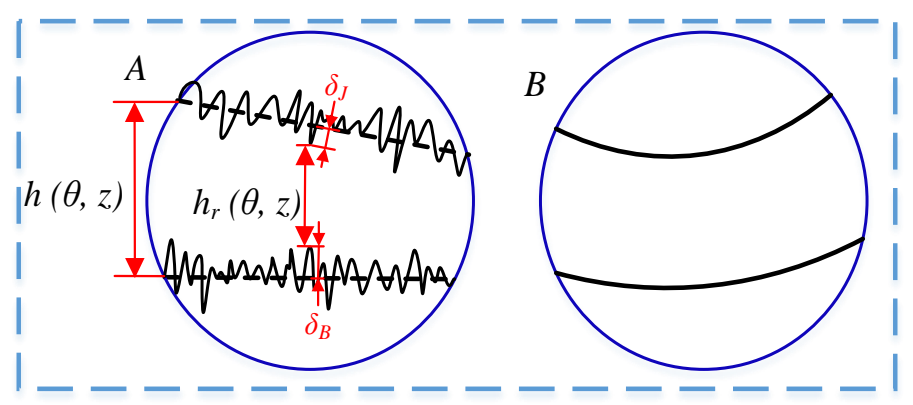

(b) Longitudinal roughness

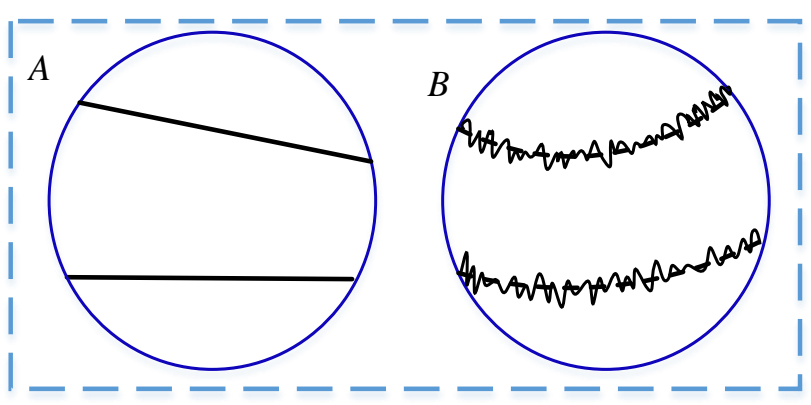

(c) Transverse roughness

Fig. 1. Geometry of a misaligned journal bearing with different surface roughness patterns.

According to the various types of rough surface patterns, the dimensionless Reynolds-type equation, 
including the one-dimensional roughness in longitudinal direction [37] as shown in Fig. 1(b), is defined in polar coordinates as

$$
\frac{\partial}{\partial \Phi}\left[\frac{\partial \bar{P}}{\partial \Phi} E\left(H^{3}\right)\right]+\frac{1}{\kappa} \frac{\partial}{\partial Z}\left[\frac{\partial \bar{P}}{\partial Z} \frac{1}{E\left(1 / H^{3}\right)}\right]=3 \frac{\partial}{\partial \Phi} E(H)
$$

where $\kappa=l / d$. $\kappa$ means the length to diameter ratio and $d$ is the diameter of bearing.

The dimensionless parameters are represented by

$$
\bar{P}=\bar{p} \frac{2 \mu \Omega r^{2}}{c^{2}}, \quad Z=\frac{z}{l / 2}, \Phi=\frac{x}{r}
$$

where $\bar{p}$ is the average pressure distribution of oil film. $\Omega$ denotes the rotating speed of fluid, which is equal to the rotating speed of the journal. $\mu$ is the fluid viscosity. $r$ means the radial of the bearing. $z$ and $x$ are the axial coordinates.

Similarly, for the pattern of surface with a transverse roughness as shown in Fig. 1(c), the stochastic Reynolds-type equation can be expressed by

$$
\frac{\partial}{\partial \Phi}\left[\frac{\partial \bar{P}}{\partial \Phi} \frac{1}{E\left(1 / H^{3}\right)}\right]+\frac{1}{\kappa} \frac{\partial}{\partial Z}\left[\frac{\partial \bar{P}}{\partial Z} E\left(H^{3}\right)\right]=3 \frac{\partial}{\partial \Phi} \frac{E\left(1 / H^{2}\right)}{E\left(1 / H^{3}\right)}
$$

where $E(*)$ denotes the expectancy operator, which is defined by

$$
E(\boldsymbol{\Xi})=\int \xi f(\xi) d \xi
$$

and $f(\xi)$ denotes the PDF of the stochastic variable $\Xi, \xi$ is a value in $\Xi$.

Generally, Christensen's model is used to analyse the average oil film pressure on the rough surface where the roughness heights follow a Gaussian distribution. However, as mentioned above, the patterns of machined surfaces have the non-Gaussian characteristics. In addition to the standard deviation and surface roughness patterns, skewness and kurtosis need to be introduced to describe the practical surface. It is well known that the skewness and kurtosis are related to the third and fourth centre moments for a random variable, respectively, and can be calculated based on the PDF of the variable. Commonly, the PDF of a random variable $\Lambda$ is difficult to obtain, but it can be considered as the nominal density function $\psi(\Lambda)$ with a zero mean and unit variance [38], so the PDF of the variable $\Lambda$ can be approximated as follows:

$$
f(\Lambda)=g_{n}(\Lambda) \psi(\Lambda)
$$

where $\psi(\Lambda)=1 / \sqrt{2 \pi} e^{-\Lambda^{2} / 2} \cdot g_{n}(\Lambda)$ is introduced to ensure that the first moment of $f(\Lambda)$ is similar 
to the PDF of the variable $\Lambda$ and can be represented by

$$
g_{n}(\Lambda)=\sum_{i=0}^{n} \varrho_{i} \chi_{i}(\Lambda)
$$

where $n$ and $\varrho_{i}$ denote the number and coefficient of the function $\chi_{i}(\Lambda)$, which means the Hermite polynomials and can be expressed by $\chi_{i}(\Lambda)=(-1)^{i} \partial^{i} \psi / \partial \Lambda^{i} / \psi(\Lambda)^{2}$. When the number $n$ is set as 4 and the mean and variance of the standardized variable $\Lambda$ expressed in Eq. (10) are zero and one, respectively, the expression of $g_{4}(\Lambda)$ is named Gram-Charlier expansion and can be represented by Eq. (11).

$$
\begin{aligned}
& \Lambda=\frac{(\delta-\bar{\delta})}{\sigma_{\delta}} \\
& g_{4}(\Lambda)=1+\frac{\gamma_{3}}{3 !} \chi_{3}(\Lambda)+\frac{\gamma_{4}}{4 !} \chi_{4}(\Lambda)
\end{aligned}
$$

Here, the three and four orders Hermite polynomials [39] are directly calculated by $\chi_{3}(\Lambda)=\Lambda^{3}+3 \Lambda$ and $\chi_{4}(\Lambda)=\Lambda^{4}-6 \Lambda^{2}+3$. In addition, the centre moment $\gamma_{j}$ is

$$
\gamma_{j}=\int_{-\infty}^{+\infty}(\Lambda-\bar{\Lambda})^{j} f(\Lambda) d \Lambda
$$

As analysed before, the variance $\sigma_{\Lambda}^{2}$ of the variable $\Lambda$, namely the second centre moment, is equal to one. That is $\sigma_{\Lambda}^{2}=\gamma_{2}=\int_{-\infty}^{+\infty}(\Lambda-\bar{\Lambda})^{2} f(\Lambda) d \Lambda=1$.

Therefore, the skewness $S_{s k}$ and kurtosis $S_{k u}$ as the standardized third and fourth centre moments can be represented by

$$
\begin{aligned}
& S_{s k}=\frac{\gamma_{3}}{\sigma_{\Lambda}^{3}}=\int_{-\infty}^{+\infty}(\Lambda-\bar{\Lambda})^{3} f(\Lambda) d \Lambda \\
& S_{k u}=\frac{\gamma_{4}}{\sigma_{\Lambda}^{4}}=\int_{-\infty}^{+\infty}(\Lambda-\bar{\Lambda})^{4} f(\Lambda) d \Lambda
\end{aligned}
$$

Submitting Eqs. (10-14) into Eq. (8), the PDF of the variable $\Lambda$ can be expressed as

$$
f(\Lambda)=\left[1+\frac{S_{s k}}{6}\left(\Lambda^{3}-3 \Lambda\right)+\frac{S_{k u}-3}{24}\left(\Lambda^{4}-6 \Lambda^{2}+3\right)\right] \frac{1}{\sqrt{2 \pi}} e^{-\frac{\Lambda^{2}}{2}}
$$

According to Eq. (15), the result can be negative or positive by setting different $S_{S k}$ and $S_{K u}$. However, the PDF of the roughness heights should be always positive. In order to satisfy the applicable conditions [38], the tuneable parameters of skewness and kurtosis should be in the range of $[-1,1]$ 
and $[0,4]$, respectively. Consequently, for the random roughness heights $\delta$, as the mean $\bar{\delta}$ is assumed to be zero to simplify the computation, the PDF with respect to the variable $\delta$ is represented by

$$
f(\delta)=\left[1+\frac{S_{s k}}{6}\left(\frac{\delta^{3}}{\sigma_{\delta}^{3}}-3 \frac{\delta}{\sigma_{\delta}}\right)+\frac{S_{k u}-3}{24}\left(\frac{\delta^{4}}{\sigma_{\delta}^{4}}-6 \frac{\delta^{2}}{\sigma_{\delta}^{2}}+3\right)\right] \frac{1}{\sqrt{2 \pi} \sigma_{\delta}} e^{\frac{-\delta^{2}}{2 \sigma_{\delta}^{2}}}
$$

Then the expectancy operator $E(*)$ of the dimensionless $\Delta$ of the roughness heights $\delta$ on the nonGaussian rough surface can be rewritten by

$$
\begin{aligned}
& E\left(H^{m}\right)=\int_{-\infty}^{+\infty}\left(H_{r}+\Delta_{s}\right)^{m} f\left(\Delta_{s}\right) d \Delta_{s} \\
& E\left(1 / H^{m}\right)=\int_{-\infty}^{+\infty} 1 /\left(H_{r}+\Delta_{s}\right)^{m} f\left(\Delta_{s}\right) d \Delta_{s}
\end{aligned}
$$

According to Christensen's methods, the stochastic Reynolds-type equations can be extended by introducing $E\left(H^{3}\right), E\left(1 / H^{3}\right), E(H)$ and $E\left(1 / H^{2}\right)$ calculated by Eqs. $(3,16-18)$. In order to obtain the average pressure distributions, the extended Eq. (4) or (6) is discretized based on the finite difference method $(\mathrm{FDM})$ with the mesh size of $(d \Phi \times d Z)$ and then the dimensionless average oil film pressure $\bar{P}$ is solved by using the successive over-relaxation (SOR) iterative method with the boundary conditions

$$
\bar{P}(\Phi, 0)=\bar{P}(\Phi, 2)=0, \quad \bar{P}(0, Z)=\bar{P}(2 \pi, Z)=0
$$

In the iterative processes, the pressure calculation is terminated by the convergence condition as follows:

$$
\frac{\sum_{i=1}^{m} \sum_{j=1}^{n}\left|\bar{P}_{i, j}^{k+1}-\bar{P}_{i, j}^{k}\right|}{\sum_{i=1}^{m} \sum_{j=1}^{n} \bar{P}_{i, j}^{k}} \leq 1 \times 10^{-6}
$$

where $m$ and $n$ denote the grid number in circumferential and axial directions. $k$ is the number of iterations.

\subsection{Performance of journal bearing}

The oil film pressure obtained can be used to compute the performance characteristics of the journal bearing and consequently incorporate the effects of non-Gaussian properties on the behaviour discussions of the misaligned journal bearing as follows.

The components of the dimensionless mean load capacity, in the circumferential and axial directions, 
can be calculated by

$$
W_{\Phi}=-\int_{0}^{2} \int_{0}^{2 \pi} \bar{P} \sin \Phi d \Phi d Z, \quad W_{z}=-\int_{0}^{2} \int_{0}^{2 \pi} \bar{P} \cos \Phi d \Phi d Z
$$

Then the total dimensionless bearing load capacity $W$ and the attitude angle $\theta$ are

$$
\begin{aligned}
& W=\widetilde{W} \frac{c^{2}}{2 \mu \Omega r^{3}}=\sqrt{W_{\Phi}^{2}+W_{z}^{2}} \\
& \theta=\arctan \left(\frac{W_{\Phi}}{W_{z}}\right)
\end{aligned}
$$

For the longitudinal roughness patterns, the dimensionless average friction force $F_{L}$ on the journal surface is given by

$$
F_{L}=\widetilde{F_{L}} \frac{c}{2 \mu \Omega r^{2}}=\int_{0}^{2} \int_{0}^{2 \pi}\left[\frac{1}{2} E(H) \frac{\partial \bar{P}}{\partial \Phi}+\frac{1}{2} E\left(\frac{1}{H}\right)\right] d \Phi d Z
$$

The dimensionless average friction force $F_{T}$, for the transverse roughness patterns, is defined as

$$
F_{T}=\widetilde{F_{T}} \frac{c}{2 \mu \Omega r^{2}}=\int_{0}^{2} \int_{0}^{2 \pi}\left\{\frac{1}{2} \frac{E\left(1 / H^{2}\right)}{E\left(1 / H^{3}\right)} \frac{\partial \bar{P}}{\partial \Phi}+\frac{1}{2}\left[4 E\left(\frac{1}{H}\right)-3 \frac{E\left(1 / H^{2}\right)}{E\left(1 / H^{3}\right)}\right]\right\} d \Phi d Z
$$

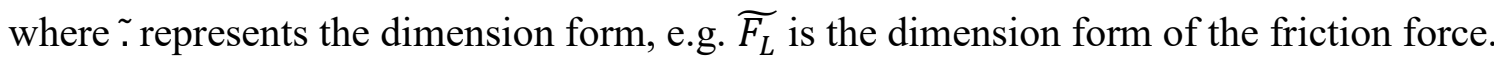

The friction coefficient $f$ can be calculated by

$$
f_{L}=\frac{c}{r} \frac{F_{L}}{W}, f_{T}=\frac{c}{r} \frac{F_{T}}{W}
$$

The dimensionless end leakage flow rates $Q_{L 1}$ and $Q_{L 2}$ from the front-end plane and the rear-end plane, considering the one- dimensional roughness in longitudinal direction, are given by

$$
Q_{L 1}=-\left.\int_{0}^{2 \pi} \frac{1}{E\left(1 / H^{3}\right)} \frac{\partial P}{\partial Z}\right|_{Z=0} d \Phi, \quad Q_{L 2}=-\left.\int_{0}^{2 \pi} \frac{1}{E\left(1 / H^{3}\right)} \frac{\partial P}{\partial Z}\right|_{Z=2} d \Phi
$$

The total dimensionless end leakage flow rate $Q_{L}$ can be represented as

$$
Q_{L}=\widetilde{Q_{L}} \frac{6 \kappa}{\Omega c r^{2}}=\left|Q_{L 1}\right|+\left|Q_{L 2}\right|
$$

Similarly, for the surface roughness in transverse, the dimensionless end leakage flow rates $Q_{T 1}$ and $Q_{T 2}$ from the front and rear end planes can be obtained as follows:

$$
Q_{T 1}=-\left.\int_{0}^{2 \pi} E\left(H^{3}\right) \frac{\partial P}{\partial Z}\right|_{Z=0} d \Phi, \quad Q_{T 2}=-\left.\int_{0}^{2 \pi} E\left(H^{3}\right) \frac{\partial P}{\partial Z}\right|_{Z=2} d \Phi
$$

Then the total dimensionless end leakage flow rate $Q_{T}$ is 


$$
Q_{T}=\widetilde{Q_{T}} \frac{6 \kappa}{\Omega c r^{2}}=\left|Q_{T 1}\right|+\left|Q_{T 2}\right|
$$

The misalignment moment at steady state is mainly caused by the misaligned journal bearing, which leads to the change of oil film pressure distribution. Two components of the moment, in the directions of circumference and axis, are expressed by

$$
M_{\Phi}=\int_{0}^{2} \int_{0}^{2 \pi} \bar{P}(Z-1) \cos \Phi d \Phi d Z, \quad M_{z}=\int_{0}^{2} \int_{0}^{2 \pi} \bar{P}(Z-1) \sin \Phi d \Phi d Z
$$

The total dimensionless misaligned moment $M$ and is given by

$$
M=\widetilde{M} \frac{2 c}{\mu \Omega r^{3} l^{2}}=\sqrt{M_{\Phi}^{2}+M_{Z}^{2}}
$$

Practically, in addition to the statically normal force for the cylindrical plain journal bearing, the vibrations perceived in the journal bearing housing, there are abundant vibrations observed in high frequency range (several $\mathrm{kHz}$ ), which is often far beyond the scope of angular frequency of the rotor. These high frequency vibrations have been understood due to a number of excitation mechanisms including the micro impulse from the solid-to-solid contact, fluid turbulences and cavitation especially for the case of high Reynolds number, background noise and so on [43, 44]. Among these effects, the high frequency vibrations mainly caused by the fluctuating force has been analysed in [45-47], which is resulted from the rough surface topographies of journal and bearing working in hydrodynamic lubrication regime. Therefore, the dynamic performance of journal bearings can be revealed by the vibration response and the change of oil film pressure distributions with respect to the non-Gaussian and misalignment of journal bearings can be verified by the various vibration response in a high frequency range, as discussed in Section 3.2.

\section{Results and discussion}

\section{$3.1 \quad$ Numerical studies}

According to the theoretical analysis above, the cylindrical plain journal bearing is utilized to discuss the performance characteristics, which are affected by the dimensionless variables such as width to diameter $\kappa$, eccentricity ratio $\varepsilon_{0}$, misalignment angle $\beta$, surface roughness $\sigma / c$, skewness $S_{S k}$ and kurtosis $S_{K u}$. The parameters used to calculate the load capacity, friction coefficient, end leakage flow rate and misalignment moment has been setup as follows: $\kappa=0.2$ and $2 ; \varepsilon_{0}=0.4,0.6,0.65,0.7,0.75$ 
and $0.8 ; \beta=0^{\circ}, 0.0015^{\circ}, 0.0050^{\circ}$ and $0.0080^{\circ} ; \sigma / c$ is chosen in the range of $[0,0.06]$ and divided into 10 equally spaced values; the values of $S_{S k}$ and $S_{K u}$, as mentioned previously, fall in the range of [-1, 1] and [0, 4], respectively. In addition, the attitude angle $\Phi_{0}$ and $\alpha$ are set as $0^{\circ}$ and $180^{\circ}$ in the following simulations. The pressure is discretised by the grids size of $41 \times 41$, which acts on the journal bearing surface with the dimensionless area of $2 \times 2 \pi$.

Fig. 2. Static performance of the bearing at the misalignment angle $\beta=0.0015^{0}$ and various eccentricity ratios: (a) load capacity (b) friction force (c) end leakage flow rate and (d) misalignment moment.

As the width to diameter $\kappa$ is 0.2 , which is usually called a narrow bearing, the static performances of the bearing have been shown in Fig. 2-Fig. 5. The effect of the increasing eccentricity ratio on the static performance of the journal bearing can be investigated in Fig. 2. Three types surface patterns are utilized to represent the smooth $(\sigma / \mathrm{c}=0)$, Gaussian $\left(S_{S k}=0, S_{K u}=3\right)$ and non-Gaussian surfaces $\left(S_{S k}=\right.$ $\left.-0.8, S_{K u}=4\right)$, respectively. The misalignment angle of journal is $0.0015^{\circ}$, which is caused by the 
deformation of shaft under the external load. The red and black lines demonstrate the results computed by the extended Reynolds-type equation with the transvers and longitudinal roughness for different $S_{S k}$ and $S_{K u}$. The load capacity $W$ is calculated by Eqs. (21-22) based on the composite Simpson integral method and the $W$ increases as the eccentricity ratio rises as shown in Fig. 2(a). In comparison with the smooth surface, the load capacity on the rough surfaces increases faster, particularly at a higher eccentricity ratio $\varepsilon_{0}$. It is because that the increasing $\varepsilon_{0}$ leads to decrease in the film thickness and consequently increase in pressure. For the surfaces with a transverse roughness, the load capacity $W$ obtained from the Gaussian rough surface is lower than that from the non-Gaussian surface as the $\varepsilon_{0}$ increases. The maximum difference of $W$ is up to $12.53 \%$ at $\varepsilon_{0}=0.8$. For the longitudinal roughness, compared with the non-Gaussian surface, the amplitude of $W$ obtained by the Gaussian surface changes from larger to smaller near the $\varepsilon_{0}=0.72$ and the maximum difference is about $8 \%$ at $\varepsilon_{0}=0.8$. In addition, when the $\kappa$ is small, the change of pressure in the axial orientation is larger than that in circumferential orientation, so the effect of the rough surface with a longitudinal roughness is greater than that with a transverse roughness. The conclusion also applies to the following analysis of the load capacity. Variations of the friction coefficient $f$ with respect to the increasing $\varepsilon_{0}$ has been shown in Fig. 2 (b) for different surface patterns. The $f$ decreases with the increasing $\varepsilon_{0}$ and drops on the smooth, Gaussian and non-Gaussian surfaces in turn, but the difference between them is not obvious. Fig. 2(c) reflects the variation of the end leakage flow rate $Q$ related to the $\varepsilon_{0}$ for different surface patterns. For each type of the surface patterns, the value of $Q$ rises as the $\varepsilon_{0}$ increases. It is noted that the $Q_{L}$ from the Gaussian surface is greater than that from the non-Gaussian surface. However, the change ratio of $Q_{L}$ from the latter is significant for the $\varepsilon_{0}$. Such effect is not obvious for $Q_{T}$. The misalignment moment $M$ increases with the increasing $\varepsilon_{0}$ for all surface patterns, particularly the $M_{L}$ for a non-Gaussian longitudinal roughness as demonstrated in Fig. 2(d). 


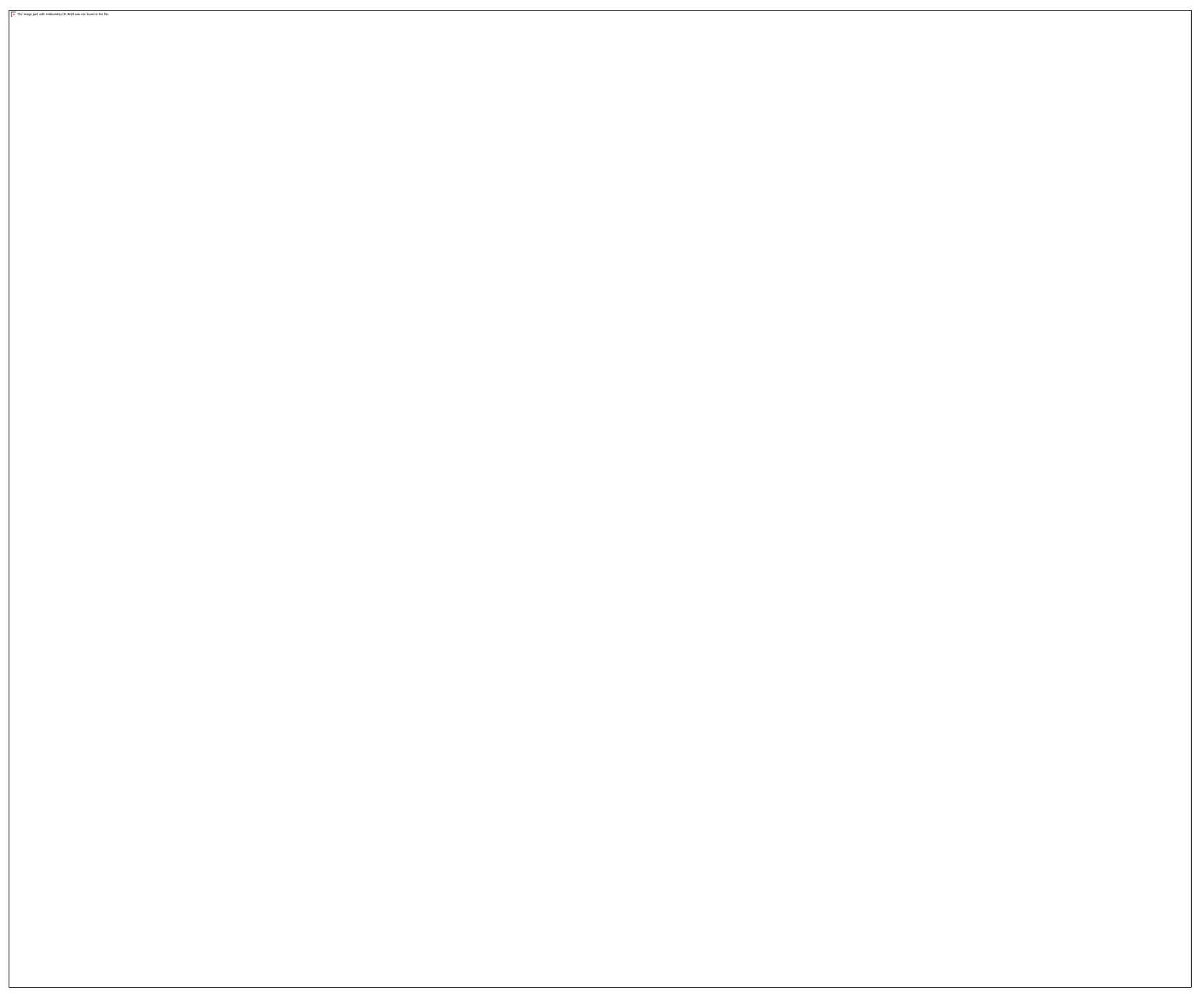

Fig. 3. Surface roughness effects on the steady-state performance of the journal bearing at $\beta=0.0015^{0}$ and $\varepsilon_{0}=0.75$ : (a) load capacity (b) friction force (c) end leakage flow rate and (d) misalignment moment.

To satisfy the journal bearing working in the hydrodynamic lubrication regime, the dimensionless roughness is setup in the range from 0 to 0.06 . The misalignment angle is set as $0.0015^{0}$ and eccentricity ratio is 0.75 . Fig. 3 shows the effects of the surface roughness on the steady-state performance characteristics of the journal bearings for the different skewness and kurtosis. The blue lines represent the results from the Gaussian rough surface, which is used to compare with that from the non-Gaussian surfaces. Variation of the $W$ with respect to the increasing roughness for different surface patterns is plotted in Fig. 3(a). According to the reason analysed above, the amplitude of the $W$ from the longitudinal roughness is higher than that from the transverse roughness. With the increasing value of $\sigma / c$, the load capacity increases for all types of rough surfaces except for the transverse roughness with $S_{S k}=0$ and $S_{K u}=0$. When the skewness is zero, the skewness effects can be ignored as the number of 
asperities on the rough surface is nearly equal to that of valleys. However, as the kurtosis is less than 3 , the high asperities and low valleys are relatively few on the rough surface resulting in the slight change in the oil film thickness, which in turn leads to the small change in the load capacity. The similar variation of the misalignment moment is represented in Fig. 3(d). Fig. 3(b) shows that the increasing roughness reduces the friction coefficient. In addition, the friction coefficient working on the non-Gaussian surface is lower compared with the Gaussian surface for the increasing surface roughness. The end leakage flow rate $Q$ related to the increasing $\sigma / \mathrm{c}$ for different surface patterns increases as investigated in Fig. 3(c).

Fig. 4. Skewness effects on the journal bearing static performance characteristics in the eccentricity ratio $\varepsilon_{0}=0.75$ (a) load capacity (b) friction force (c) end leakage flow rate and (d) misalignment moment. 


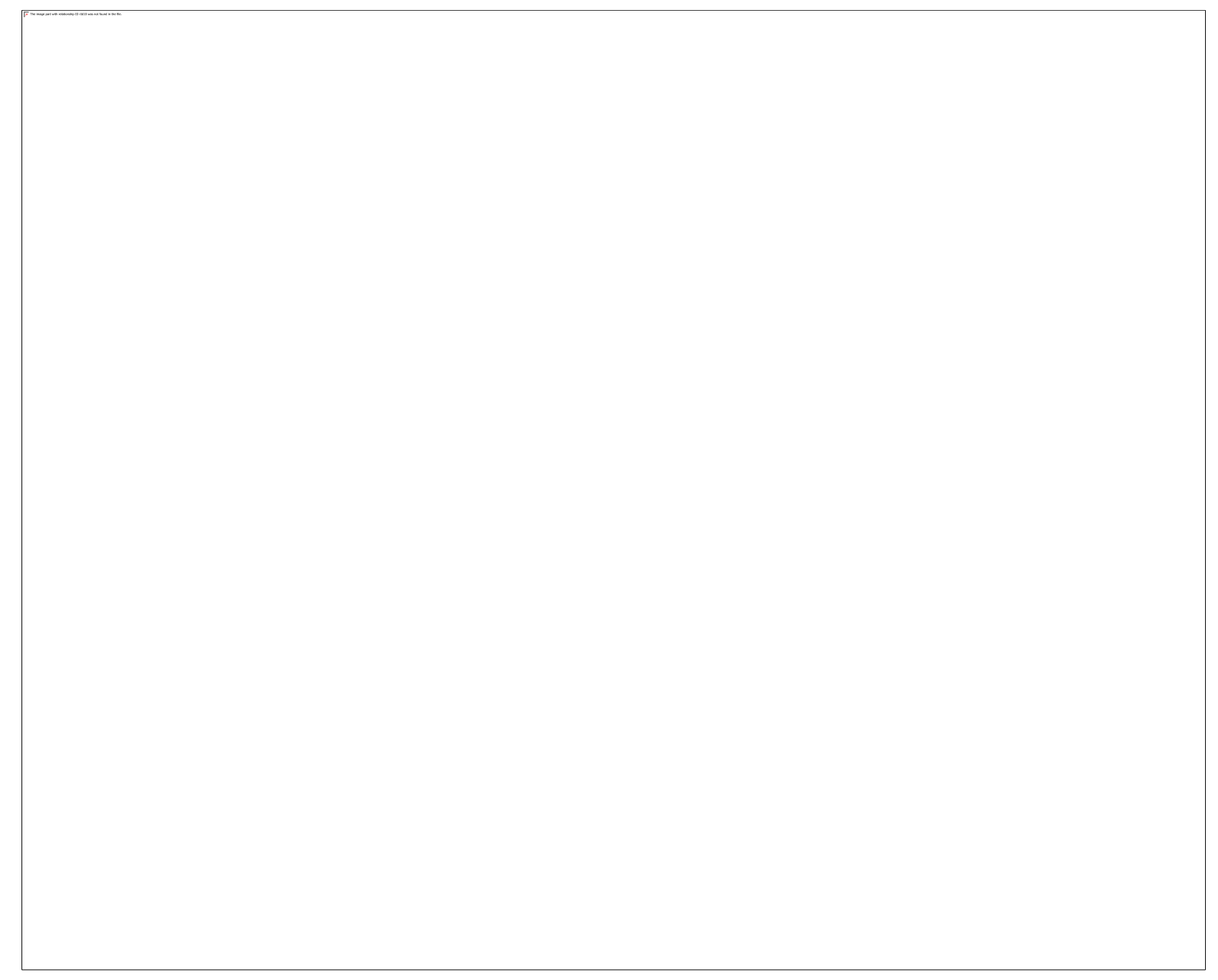

Fig. 5. Kurtosis effects on the bearing static performance at $\varepsilon_{0}=0.75$ with the different misalignment angles: (a) load capacity (b) friction force (c) end leakage flow rate and (d) misalignment moment.

Fig. 4 represents the influence of the skewness on the static performance of hydrodynamic journal bearing with the misalignment angle of $0.0015^{\circ}$ and $0.0050^{\circ}$. The dimensionless surface roughness is set as $\sigma / \mathrm{c}=0.06$ and the kurtosis is 3 . In addition, the aligned journal is introduced to compare the steady-state performance with the misaligned journal for the various skewness as the blue lines plotted in each of the subgraphs. The load capacity decreases with the skewness ranging from -1 to 1 for the different misalignment angles as investigated in Fig. 4(a). Clearly, comparison with the aligned journal, the variation of the $W$ is not obvious at the misalignment angle of $0.0015^{\circ}$. However, as the misalignment angle is $0.0050^{\circ}$, the maximum difference is about $5.52 \%$ at the $S_{S K}=-1$, which demonstrates that the effect of the non-Gaussian rough surface is significant at a large misalignment. The change in the friction coefficient is an inverse trend of the load capacity as shown in Fig. 4(b) and 
about $4.19 \%$ difference between the misaligned angles of $0.0050^{\circ}$ and $0^{\circ}$ at $S_{S K}=-1$. The end leakage flow rate and misalignment moment decrease with the increasing skewness for the misaligned journal, as shown in Fig. 4(c) and (d). Similarly, the maximum difference compared with the aligned journal appears at $S_{S K}=-1$ with a larger misalignment angle of $0.0050^{\circ}$. For these results, it can be explained that the rough surface with a negative skewness leads to the oil film thickness decrease and consequently result in the oil film pressure increasing.

Fig. 5 depicts the effect of the kurtosis $S_{K u}$ on the static performance of the journal bearing for different misalignment angles at the eccentricity ratio of 0.75 and the dimensionless roughness of 0.06 . The load capacity with respect to the increasing $S_{K u}$ is in decline for different misalignment angles as shown in Fig. 5(a). However, the change of the $W$ for the transverse roughness raises linearly with the increasing kurtosis, particularly at the misalignment angle of $0.0050^{\circ}$. The influence of $S_{K u}$ on the friction coefficient $f$ is demonstrated in Fig. 5(b). The increased $S_{K u}$ results the dropped $f$ for the longitudinal roughness patterns, but the raised $f$ for the transverse roughness patterns. Fig. 5(c) represents that the end leakage flow rate decreases as the value of $S_{K u}$ increases. However, the change is not obvious between the different misaligned angles. With the increasing misalignment angle, the misalignment moment $M$ increases as investigated in Fig. 5(d), but it has little impact on the $M$ with the various $S_{K u}$.

As the width to diameter $\kappa$ is 2, usually called a long bearing, the results of the load capacity, friction coefficient, end leakage flow rate and misalignment moment of the journal bearing have been shown in Fig. 6 -Fig. 9. The change of the static performances of the journal bearing with respect to the various $\varepsilon_{0}$ for the transverse roughness pattern is still greater than that for the longitudinal roughness pattern as investigated in Fig. 6(a), (b), (c) and (d). The dimensionless surface roughness is set as $\sigma / c=0.06$ and the misalignment angle $0.0015^{\circ}$. With the $\varepsilon_{0}$ increasing from 0.4 to 0.8 , the values of the $W, Q$ and $M$ increase, but the value of the $f$ decreases. In addition, the non-Gaussian rough surface results a larger load capacity compared with the Gaussian surface as the value of $\varepsilon_{0}$ is over 0.6 for the misaligned journal bearing. The maximum difference is about $17.84 \%$ at $\varepsilon_{0}=0.8$ for the transverse roughness pattern. The variation of the misalignment moment $M$ is similar to the load capacity and the maximum difference is $19.81 \%$. 


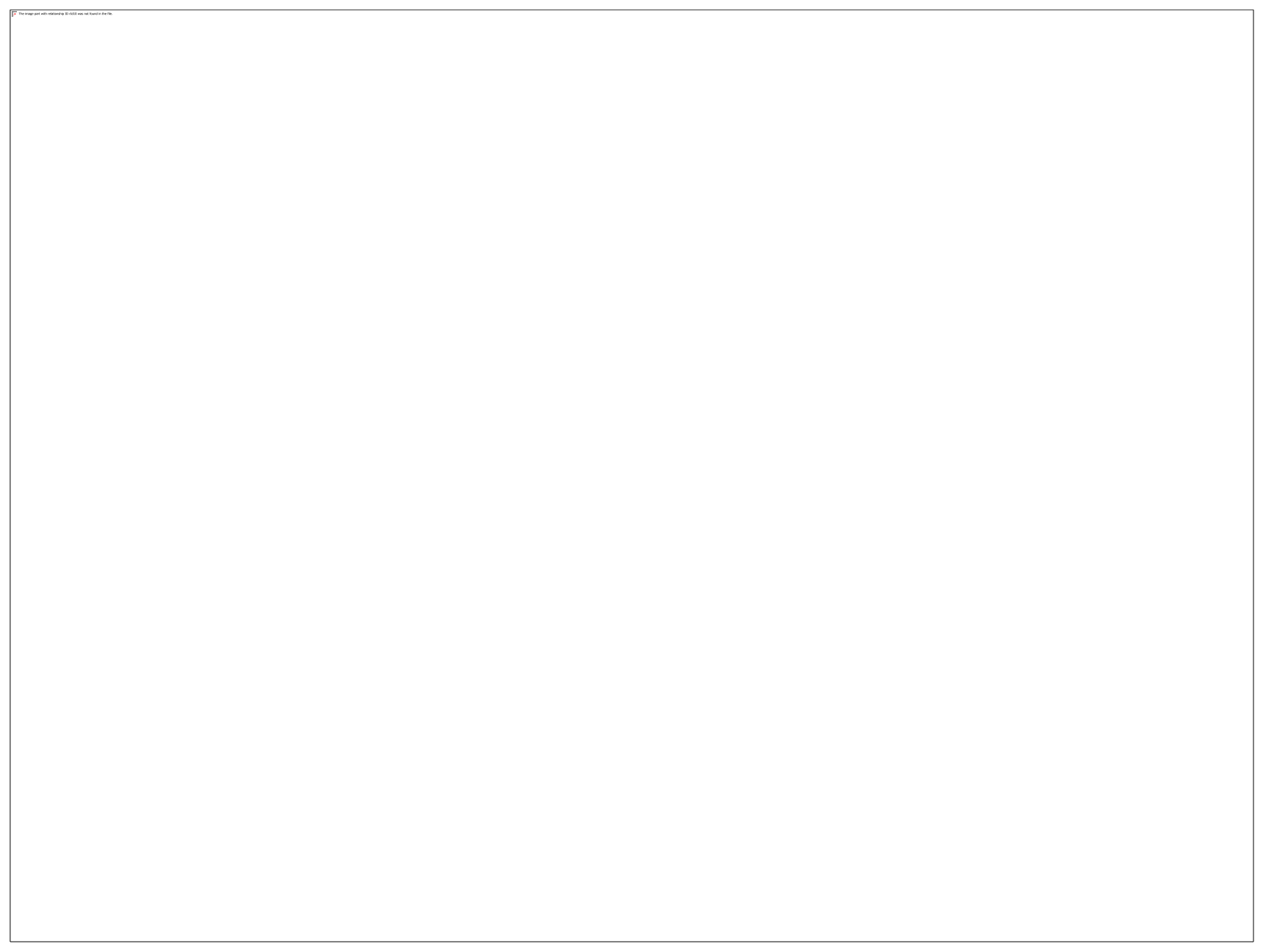

Fig. 6. Static performance of the bearing with the $\beta=0.0015^{0}$ and $\sigma / c=0.06$ at various eccentricity ratios: (a) load capacity (b) friction force (c) end leakage flow rate and (d) misalignment moment.

Fig. 7 demonstrates the effect of the dimensionless roughness $\sigma / c$ on the static performance of the misaligned journal bearing for the different surface patterns with the same misalignment angle and eccentricity ratio. The load capacity $W$, end leakage flow rate $Q$ and misalignment moment $M$ rise with respect to the increased $\sigma / c$. However, the change is reverse for the friction coefficient $f$. When the value of $\sigma / \mathrm{c}$ is less than 0.0467 , the effect of the kurtosis is significant for the $W$. However, the negative skewness mainly affects the load capacity when the $\sigma / c$ is over 0.0467 , as illustrated in Fig. 7(a). The kurtosis is critical to the rise of the end leakage flow rate compared with the various skewness with the increased $\sigma / \mathrm{c}$ as demonstrated in Fig. 7(c). As mentioned above, the misalignment moment is calculated based on the pressure, which is similar to the load capacity change, so that the increase of $M$ is significant with the negative skewness $S_{S k}=-0.8$ as shown in Fig. 7(d).

In order to analyse the skewness effects on the static performance of the long journal bearing, the 
eccentricity ratio is set as 0.75 and the kurtosis 3, the results are shown in Fig. 8. With the increased skewness for the different misalignment angles, the $W, Q$ and $M$ decrease as represented in Fig. 8(a), (c) and (d), but for the $f$, it increases with the raising skewness, as shown in Fig. 8(b). In addition, the effect of the skewness is more obvious at the misalignment angle of $0.0050^{\circ}$ compared with the misalignment angles of $0^{\circ}$ and $0.0015^{\circ}$ for the load capacity and the misalignment moment. However, for the friction coefficient and end leakage flow rate, the variation is not visible between different misalignment angles.

Fig. 7. Surface roughness effects on the steady-state performance of the bearing at $\beta=0.0015^{0}$ and $\varepsilon_{0}=0.75$ : (a) load capacity (b) friction force (c) end leakage flow rate and (d) misalignment moment. 


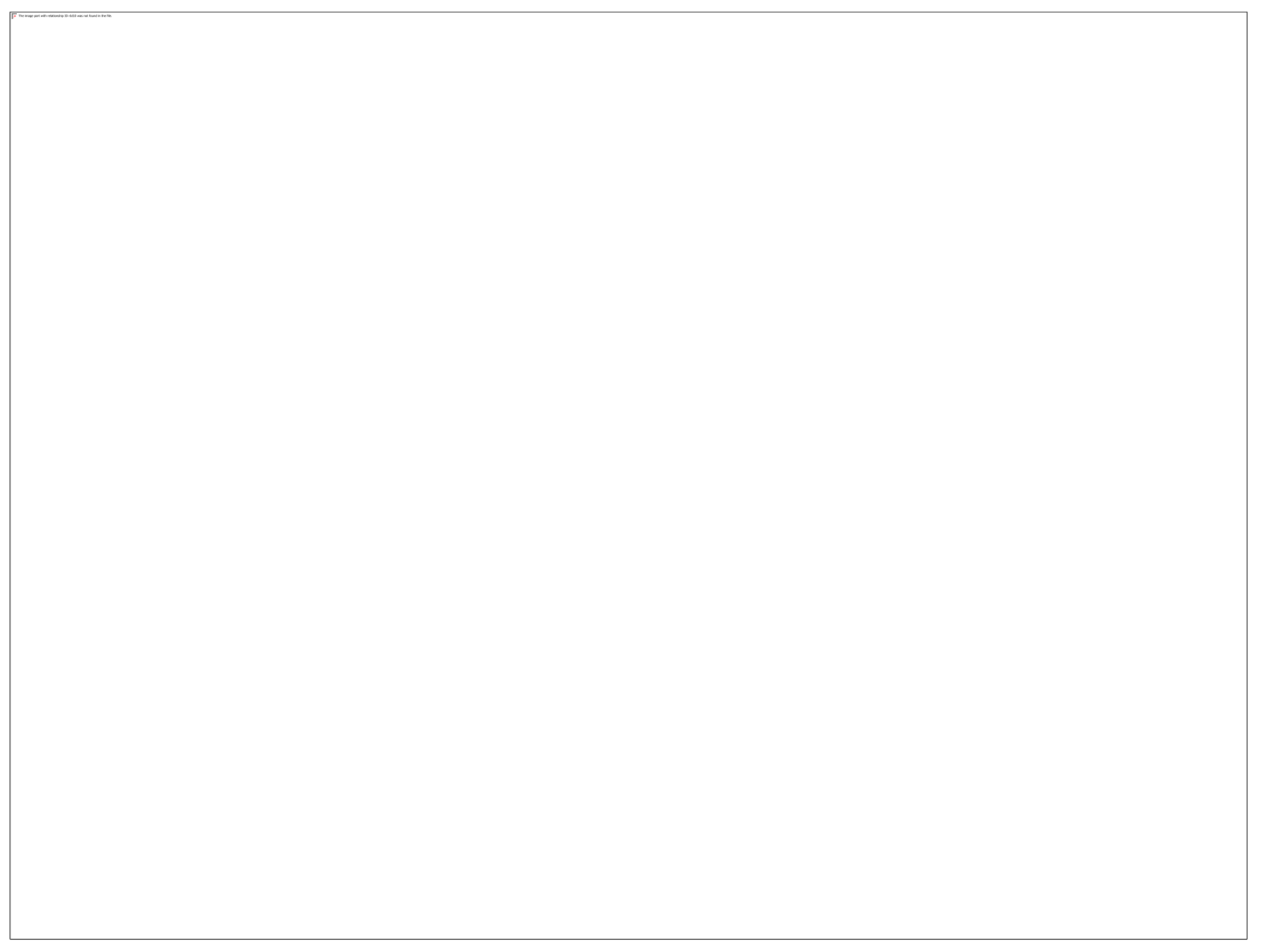

Fig. 8. Skewness effects on the bearing static performance at the $\varepsilon_{0}=0.75$ with different misalignment angles: (a) load capacity (b) friction force (c) end leakage flow rate and (d) misalignment moment.

Fig. 9 illustrates the kurtosis effects in the analysis of bearing static performances for the different misalignment angles with the same skewness $S_{S k}=0$ and eccentricity $\varepsilon_{0}=0.75$. The load capacity $W$ and misalignment moment $M$ rise with the increase in the kurtosis, especially with the misalignment angle of $0.0050^{\circ}$ as shown in Fig. 9(a) and (d). Inversely, the falling friction coefficient is plotted in Fig. 9(b) for the increasing kurtosis. Fig. 9(c) depicts that the end leakage flow rate $Q$ drops dramatically as the kurtosis increases, but there is no obvious difference between the different misalignment angles. The reason may be that the pressure gradient in axial direction is small for a long bearing which is in turn the slight change in the end leakage flow rate based on the Eqs. $(27,29)$. 


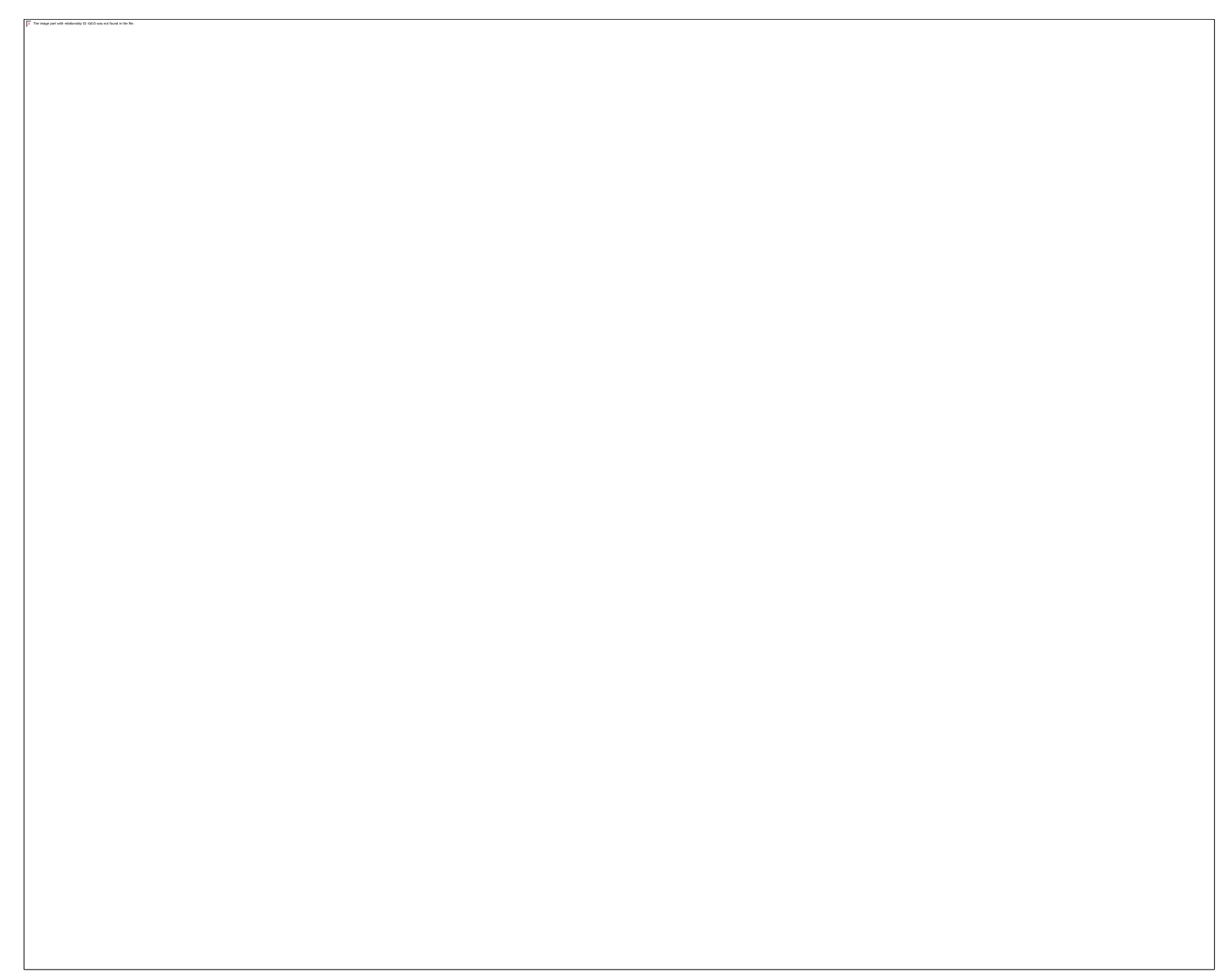

Fig. 9. Kurtosis effects on the static performance of the bearing at $\varepsilon_{0}=0.75$ with the different misalignment angles: (a) load capacity (b) friction force (c) end leakage flow rate and (d) misalignment moment.

\subsection{Experimental analysis}

To understand intuitively the effects of the skewness and kurtosis on the dynamic performance of the misaligned journal bearing, it is crucial to evaluate the relationship between the surface roughness parameters and local pressure distributions. Therefore, the relative simulations and experiments are analyzed as following.

According to numerical studies, the pressure distributions of oil film under different conditions are investigated in Fig. 10 and the maximum dimensionless pressure is noted in the figure. The eccentricity ratio for the analysis is set as 0.75 and the width to diameter is 2.14 . Fig. 10(a) depicts the pressure distribution of the oil film with an aligned journal and smooth surface. The distribution of pressure is symmetric along the $\mathrm{z}$ axis and the maximum dimensionless pressure is $P_{\max }=0.8820$. The journal with a misalignment angle of $0.008^{\circ}$ is utilized to analyse the pressure distribution is shown in Fig. 10(c). The distribution of pressure in $\mathrm{z}$ axial is asymmetric and the maximum dimensionless pressures 
is 1.0236, which is higher than that in Fig. 10(a). Comparison with Fig. 10(b) and (d), the distributions of oil film pressure are simulated with the same misalignment angle of $0.0015^{\circ}$ and the dimensionless roughness of 0.006 . The asymmetric distribution of pressure is mainly caused by the misalignment, but the change of the $\bar{P}_{\max }$ is resulted from the variation of rough surface patterns. It can be concluded that the non-Gaussian rough surface leads to a more local pressure caused by the local lubricant film thickness decreases. In addition, the effect of skewness and kurtosis exceeds that of the misalignment by comparing Fig. 10(c) and (d). The non-Gaussian rough surface with a lower misalignment angle leads to the maximum pressure increase about $36.8 \%$.

The journal bearing test rig is developed to carry out the relative experiments to verify the effects of non-Gaussian properties and misalignments on the oil film local pressure distributions. The surface topographies of self-aligning journal bearings (SA35M, $d=35 \mathrm{~mm}, l=75 \mathrm{~mm}$ ) are measured by the Alicona Infinite Focus microscope and then installed on the test rig respectively, as shown in Fig. 11. For the long journal bearing $(\kappa=l / d=2.14)$, the following experiments are operated at a constant speed of $900 \mathrm{rpm}$ controlled by the AC motor. The various external radial loads ( $3 \mathrm{bar}=0.3 \mathrm{MPa}, 9$ bar $=0.9 \mathrm{MPa}$ and $12 \mathrm{bar}=1.2 \mathrm{MPa}$ ) are applied on the rotating shaft by the hydraulic device to obtain the varying misaligned angles, which can be defined by $\beta=W L^{2} / 16 E J$. Here, $L, E$ and $J$ denote the length, modulus of elasticity and inertial moment of cross-section of the rotating shaft, respectively. To illustrate the lubrication regimes of the journal bearing operation, Hersey number is introduced by the formulas $H e=\mu \Omega R / P$, where $P$ is the unite load and $\rho$ is the density of the lubricant HV32. For the different operating conditions, the calculated result of the Hersey number is $\mathrm{He} \geq 2.0556 \times 10^{-7}$ $\mathrm{m}$. Therefore, the tested journal bearing can be considered to operate in the hydrodynamic lubricant regime. Meanwhile, the accelerometers with a wide frequency response up to $10 \mathrm{kHz}$ are used to obtain the vibration signals from the journal bearing housing with a sampling rate of $96 \mathrm{kHz}$.

The spectral response of the vibration with respect to the healthy and scratched surfaces of journal bearings has been plotted in Fig. 12(a) and (b), which are operated at different radial loads. Figure 12(a) investigates that a greater misaligned angle results in the raising amplitude of vibration response in the range of $3.8 \mathrm{kHz}$ to $4.5 \mathrm{kHz}$ and the similar change for the slightly scratched journal bearing is shown in Fig. 12(b). Refer to [40], the oil film pressure perturbation in the hydrodynamic lubrication regime is mainly caused by the fluid-asperity interaction and leads to the resonant response in a wide 
and high frequency band. The more misalignment leads to the local oil film pressure increases and consequently in turn results in a greater amplitude of the resonant vibration. In addition, the spectral distribution is different when the surface roughness is almost the same but other surface roughness parameters are different. Therefore, the non-Gaussian properties should be considered, particularly for monitoring the early fault of the bearing surface. Generally, when the misaligned angle is larger, the local oil film pressure is higher, which leads to the strong fluctuating pressure. Practically, according to Fig. 12(c), the amplitude of vibration responses from the healthy bearing with a larger misaligned journal (load 12 bar), is clearly greater than that from the scratched bearing with a lower load 3 bar. Therefore, in comparison with the misalignment impact, the non-Gaussian properties play a main role in changing vibration response amplitudes. Moreover, in comparison with the misalignment impact, the non-Gaussian properties play the critical roles on the change of the vibration response amplitude as shown in Fig. 12(c). These trends of tested results are consistent with the oil film pressure simulated by the derived theoretical model.
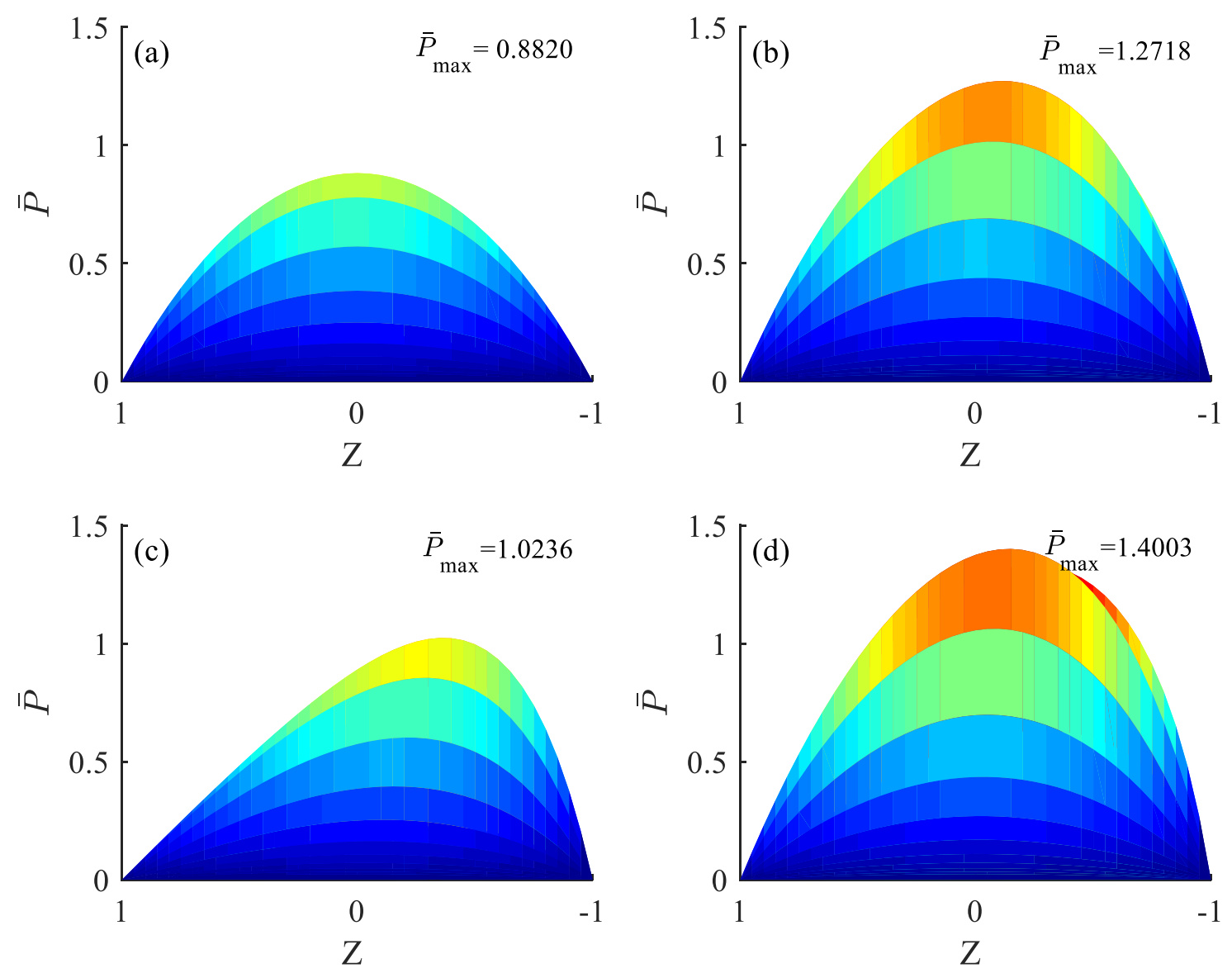

Fig. 10. Oil film pressure at different conditions: (a) $\beta=0^{\circ}$ and $\sigma=0$ (b) $\beta=0.0015^{\circ}, \sigma / c=0.06, S_{S k}=0$ and $S_{K u}=3$ (c) $\beta=0.008^{\circ}$ and $\sigma=0$ (d) $\beta=0.0015^{\circ}, \sigma / c=0.06, S_{S k}=-0.8$ and $S_{K u}=4$. 


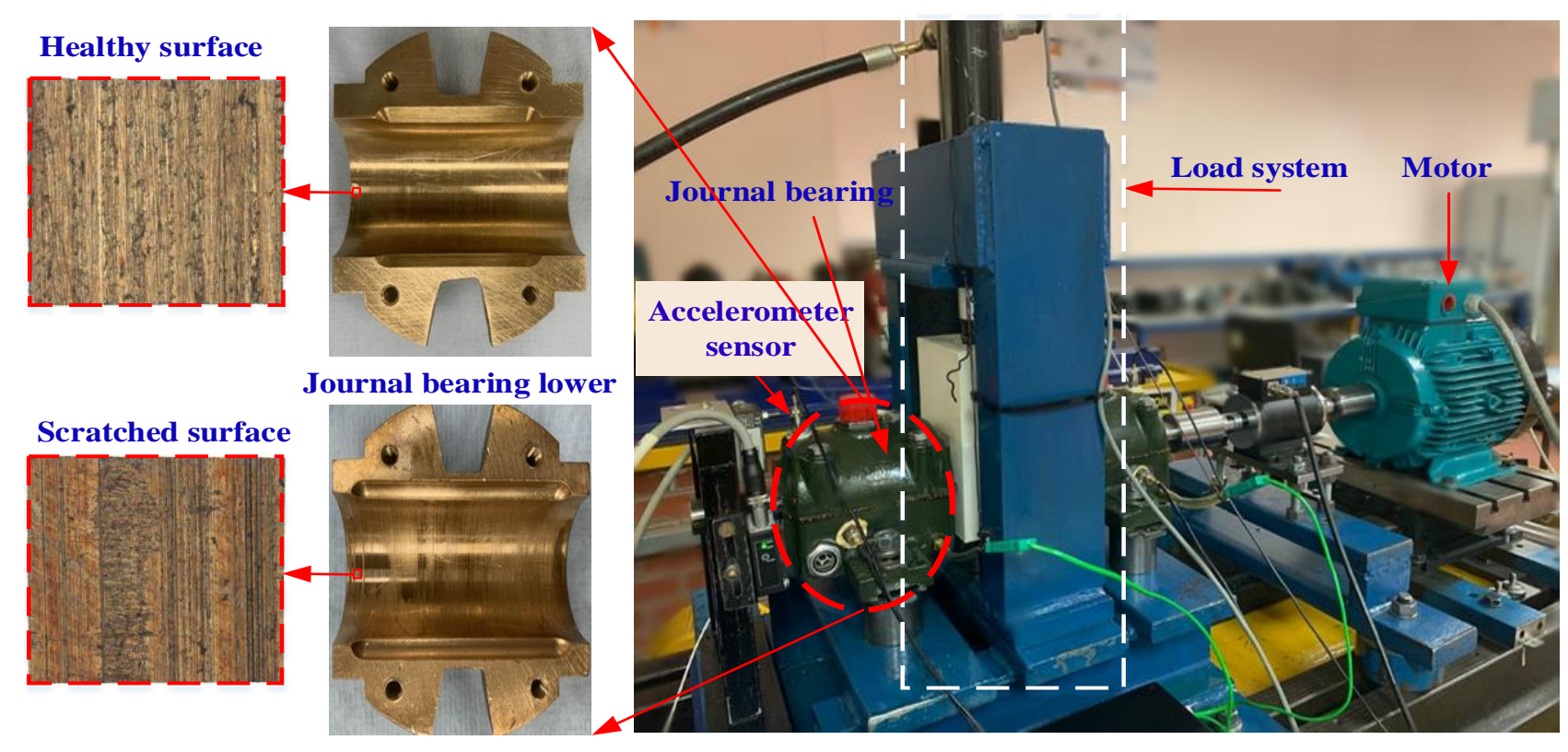

Fig. 11. Journal bearing test rig.
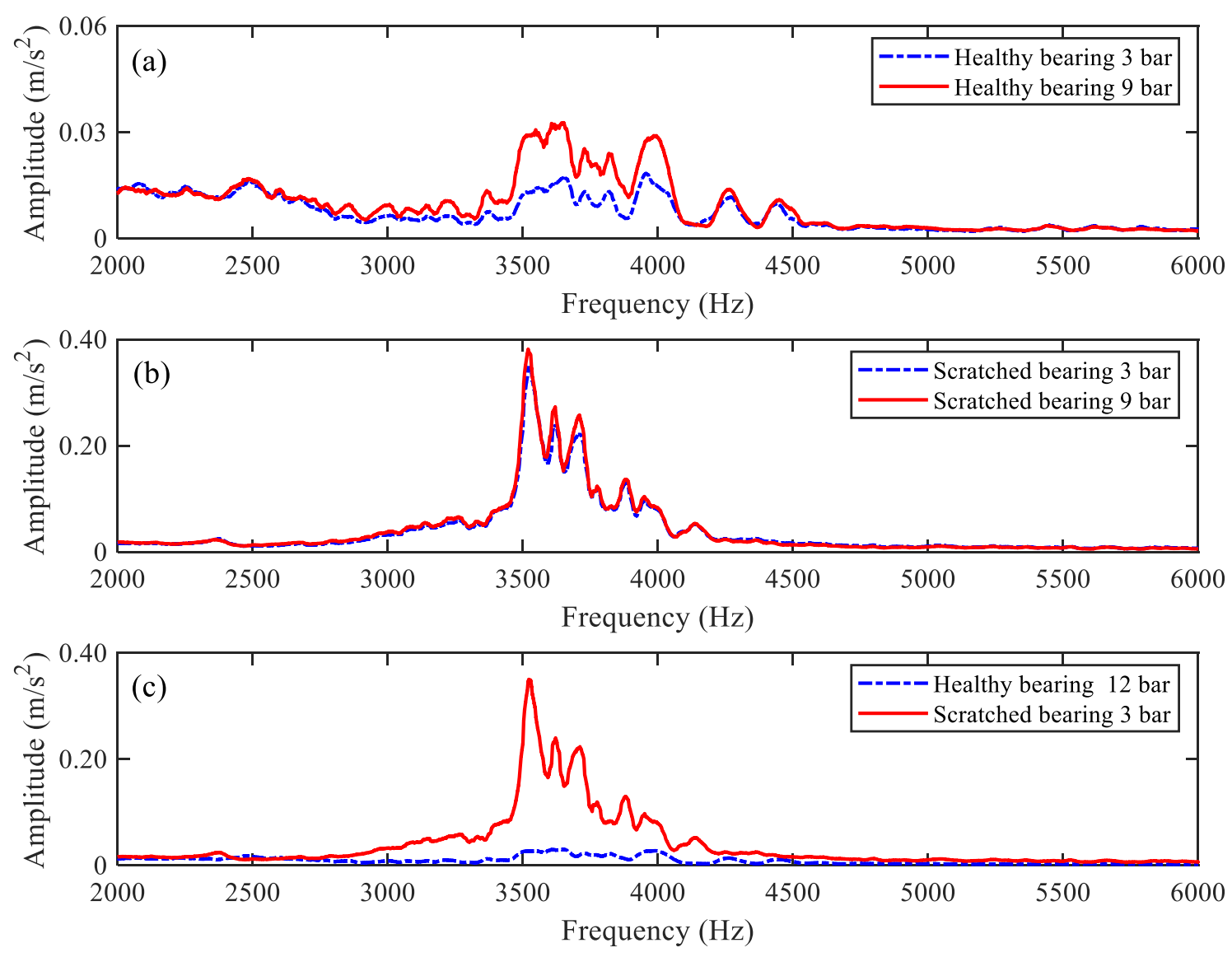

Fig. 12. Spectra distributions of vibration response from the journal bearing housing with a constant rotating speed of $900 \mathrm{rpm}$ and different surface topographies: (a) a healthy surface ( $S_{a}=0.65 \mu \mathrm{m}, S_{S k}=0.086$ and $\left.S_{K u}=2.68\right)$, (b) scratched surface $\left(S_{a}=0.60 \mu \mathrm{m}, S_{S k}=-0.491\right.$ and $\left.S_{K u}=3.996\right)$, and (c) load 12 bar for the healthy bearing and load 3 bar for the scratched bearing. 


\section{Conclusion}

In this study, the stochastic Reynolds-type equation is extended by introducing the Gram-Charlier expansion to incorporate the skewness and kurtosis effects in the analysis of the load capacity, friction coefficient, end leakage flow rate and misalignment moment of the misaligned journal bearing working in hydrodynamic lubrication regime. According to the above comparisons of variables such as $\varepsilon_{0}, \sigma / c$, $S_{S k}$ and $S_{K u}$ for the different width to diameter ratios $\kappa$ and misalignment angles $\beta$, several conclusions can be drawn as follows:

(1) Compared with the Gaussian rough surface, the non-Gaussian aspect of the rough surface increases the load capacity about $12.53 \%, 17.42 \%$ and decreases the friction coefficient about $8.96 \%$, $13.36 \%$ for $\kappa=0.2$ and $\kappa=2$ at the eccentricity ratio $\varepsilon_{0}=0.8$.

(2) For a short journal bearing, the roughness in longitudinal orientation significantly affects the static performance of the misaligned journal bearing, whereas for a long journal bearing, the influence of transverse roughness on the misaligned journal bearing performance is obvious.

(3) The negative skewness mainly rises the load capacity of the misaligned journal bearing, particularly at $\beta=0.0050^{\circ}$, and the increasing kurtosis decreases the end leakage flow rate.

(4) The effects of non-Gaussian rough surface on the performance of journal bearings are far greater than that misalignment verified by the simulations and experiments.

\section{Acknowledgement}

This research is supported by the National Natural Science Foundation of China (Grant no. 51875166, 51705127) and the Tribology Science Fund of the State Key Laboratory of Tribology (Grant no. SKLTKF18A05).

\section{Nomenclature}

c

$e_{0} \quad$ eccentricity at the axial mid-plane of the bearing

$\Phi_{0} \quad$ attitude angle at the axial mid-plane of the bearing

$l$

$d$

$r$

$\kappa$

radial clearance

bearing width

diameter of bearing

radial of the bearing

length to diameter ratio 


$\begin{array}{ll}z, x & \text { axial coordinates } \\ \beta & \text { misalignment angle } \\ h & \text { local oil film thickness } \\ h_{r} & \text { oil film thickness considering the journal misalignment } \\ \delta_{J} & \text { roughness amplitudes of journal surface } \\ \delta_{B} & \text { roughness amplitudes of bearing surfaces } \\ \delta & \text { nominal roughness height } \\ \sigma & \text { standard deviation of the roughness height } \\ H & \text { dimensionless local oil film thickness } \\ H_{r} & \text { dimensionless oil film thickness considering the journal misalignment } \\ \varepsilon_{0} & \text { dimensionless eccentricity } \\ \Delta & \text { dimensionless nominal roughness height } \\ \bar{P} & \text { dimensionless average pressure distribution of oil film } \\ \Omega & \text { rotating speed of fluid } \\ \mu & \text { fluid viscosity } \\ E(*) & \text { expectancy operator } \\ f(*) & \text { PDF of the stochastic variable } \\ \psi(*) & \text { nominal density function } \\ \chi_{i} & \text { Hermite polynomials } \\ \gamma_{j} & \text { centre moment } \\ \Lambda & \text { standardized variable } \\ S_{s k} & \text { skewness, standardized third centre moments of a random variable } \\ S_{k u} & \text { kurtosis, standardized fourth centre moments of a random variable } \\ W & \text { dimensionless bearing load capacity } \\ f_{L} & \text { dimensionless average friction coefficient for longitudinal roughness } \\ f_{T} & \text { dimensionless average friction coefficient for transverse roughness } \\ Q_{L} & \text { dimensionless end leakage flow rate for longitudinal roughness } \\ Q_{T} & \text { dimensionless end leakage flow rate for transverse roughness } \\ M & \text { dimensionless misaligned moment }\end{array}$

Reference

[1] H.Urreta, G. Aguirre, P. Kuzhir, L.N. Lopez de Lacalle, Actively lubricated hybrid journal bearings based on magnetic fluids for high-precision spindles of machine tools, J Intel Mat Syst Str 30(15) (2019) 2257-2271.

[2] H.Urreta, G. Aguirre, P. Kuzhir, L.N. Lopez de Lacalle, Seals Based on Magnetic Fluids for High Precision Spindles of Machine Tools, Int J Precis Eng Man 19(4) (2018) 495-503.

[3] L.B. Visnadi, H.F. Castro, Influence of bearing clearance and oil temperature uncertainties on the stability threshold of cylindrical journal bearings, Mech Mach Theory 134 (2020) 57-73.

[4] X. Wang, L. Zhou, M. Huang, X. Yue, Q. Xu, Numerical investigation of journal misalignment on the static and dynamic characteristics of aerostatic journal bearings, Measurement 128 (2018) 314-324.

[5] J. Ma, H. Zhang, Z. Shi, V. Kontogiorgos, F. Gu, D.A. Ball. Vibration analysis of journal bearings under water contaminated lubrication, In 24th International Conference on Automation and Computing (ICAC) (2018) 1-6. 
[6] W. Zhou, N. Qiu, L. Wang, B. Gao, D. Liu, Dynamic analysis of a planar multistage centrifugal pump rotor system based on a novel coupled model, J Sound Vib 434 (2018) 237-260.

[7] M.A. Hili, S. Bouaziz, M. Maatar, T. Fakhfakh, M. Haddar, Hydrodynamic and elastohydrodynamic studies of a cylindrical journal bearing, J Hydrodyn 22 (2010) 155-163.

[8] C. Fu, Y. Xu, Y. Yang, K. Lu, F. Gu, A.D. Ball, Response analysis of an accelerating unbalanced rotating system with both random and interval variables, J Sound Vib 466 (2020) 15047.

[9] S. MaKee, T. McKee, Pressure distribution in the oil film of journal bearing, Trans ASME 54 (1932) 149-165.

[10] G. Dubois, H.H. Mabie, F.W. Ocvirk, Experimental investigation of oil film pressure distribution for misaligned plain bearings, 1951 .

[11] Pinkus, S. Bupara, Analysis of misaligned grooved journal bearings, J Lubr Technol (1979) 503-509.

[12] R. Buckholz, J.F. Lin, The effect of journal bearing misalignment on load and cavitation for non-Newtonian lubricants. J Tribol (1986) 645-654.

[13] D. Vijayaraghavan, Jr.T. Keith, Effect of cavitation on the performance of a grooved misaligned journal bearing, Wear 134(2) (1989) 377-397.

[14] D. Vijayaraghavan, Jr.T. Keith, Analysis of a finite grooved misaligned journal bearing considering cavitation and starvation effects. J Tribol (1990) 60-67.

[15] J. Sun, G. Changlin, Hydrodynamic lubrication analysis of journal bearing considering misalignment caused by shaft deformation, Tribol Int 37(10) (2004) 841-848.

[16] F. Lv, C. Jiao, N. Ta, Z. Rao, Mixed-lubrication analysis of misaligned bearing considering turbulence, Tribol Int 119 (2018) 19-26.

[17] Ebrat, Z.P. Mourelatos, N. Vlahopoulos, K. Vaidyanathan, Calculation of journal bearing dynamic characteristics including journal misalignment and bearing structural deformation, Tribol Trans 47(1) (2004) 94-102.

[18] N. Ram, S.C. Sharma, Influence of wear on the performance of hole-entry hybrid misaligned journal bearing in turbulent regime, Ind Lubr Tribol 2014.

[19] G. Xu, J. Zhou, H. Geng, M. Lu, L. Yang, L. Yu, Research on the static and dynamic characteristics of misaligned journal bearing considering the turbulent and thermohydrodynamic effects, J Tribol 137(2) (2015).

[20] X. Zhang, Z. Yin, D. Jiang, G. Gao, Y. Wang, X. Wang, Load carrying capacity of misaligned hydrodynamic water-lubricated plain journal bearings with rigid bush materials, Tribol Int 99 (2016) 1-13.

[21] S. Das, S.K. Guha, Numerical analysis of steady-state performance of misaligned journal bearings with turbulent effect, J Braz Soc Mech Sci Eng 41(2) (2019) 81.

[22] L. Abdel-Latif, M. Mokhtar, Misalignment effects on hydrodynamicaly lubricated journal bearings with rough surfaces, Wear 128(3) (1988) 225-237.

[23] S. Guha, Analysis of steady-state characteristics of misaligned hydrodynamic journal bearings with isotropic roughness effect, Tribol Int 33(1) (2000) 1-12.

[24] S.C. Sharma, S. Jain, T. Nagaraju, Combined influence of journal misalignment and surface roughness on the performance of an orifice compensated non-recessed hybrid journal bearing, Tribol Trans 45(4) (2002) 457-63.

[25] J. Sun, M. Deng, Y. Fu, C. Gui, Thermohydrodynamic lubrication analysis of misaligned plain journal bearing with rough surface, J Tribol 132(1) (2010).

[26] J. Sun, X. Zhu, L. Zhang, X. Wang, C. Wang, H. Wang, X. Zhao, Effect of surface roughness, viscosity-pressure relationship and elastic deformation on lubrication performance of misaligned journal bearings, J Lubr Technol $66(3)$ (2014) 337-345.

[27] S. Zhu, J. Sun, B. Li, G. Zhu, Thermal turbulent lubrication analysis of rough surface journal bearing with journal misalignment, Tribol Int 144 (2020) 106-109.

[28] J. Ma, H. Zhang, F. Chu, Z. Shi, F. Gu, D.A. Ball, Modelling Acoustic Emissions induced by dynamic fluidasperity shearing in hydrodynamic lubrication regime, Tribol Int 153 (2020) 106590.

[29] S.P.L. Kumar, Measurement and uncertainty analysis of surface roughness and material removal rate in micro turning operation and process parameters optimization, Measurement 140 (2019) 538-547. 
[30] C. Fu, X. Ren, Y. Yang, Y. Xia, W. Deng, An interval precise integration method for transient unbalance response analysis of rotor system with uncertainty, Mech Syst Signal Process 107 (2018) 137-148.

[31] B. González-Ciordia, B. Fernández, G. Artola, M. Muro, A. Sanz, L.N. Lopez de Lacalle, Failure-Analysis Based Redesign of Furnace Conveyor System Components: A Case Study. Metals 9(8) (2019) 816.

[32] J.I. McCool, Manufacture, Non-Gaussian effects in microcontact, Int J Mach Tools Manu 32(1-2) (1992) 115123.

[33] C. A. Kotwal, B. Bhushan, Contact analysis of non-Gaussian surfaces for minimum static and kinetic friction and wear, Tribol Trans 39(4) (1996) 890-898.

[34] V. Bakolas, Numerical generation of arbitrarily oriented non-Gaussian three-dimensional rough surfaces, Wear 254 (5-6) (2003) 546-554.

[35] T. Kim, B. Bhushan, Y. Cho, The contact behavior of elastic/plastic non-Gaussian rough surfaces, Tribol Lett 22(1) (2006) 1

[36] Y.J. Cho, T.W. Kim, Y.P. Koo, Effect of Kurtosis on the flow factors using average flow model, JSME Int J, Ser C 47(1) (2004) 429-434.

[37] W.Z. Wang, H. Chen, Y.Z. Hu, H. Wang, Effect of surface roughness parameters on mixed lubrication characteristics, Tribol Int 39(6) (2006) 522-527.

[38] J. Li, G. Zhang, Y. Huang, R. Chen, S. Yan, H. Cao, Influence of non-Gaussian-distributed surface roughness on the static performance of slider bearings, Tribol Trans 60(4) (2017) 739-752.

[39] Y. Wang, Y. Liu, Z. Wang, Y. Wang, Surface roughness characteristics effects on fluid load capability of tilt pad thrust bearings with water lubrication, Friction 5(4) (2017) 392-401.

[40] H. Christensen, Stochastic models for hydrodynamic lubrication of rough surfaces, Proc Inst Mech Eng 184(1) (1969) 1013-26.

[41] E. Jondeau, M. Rockinger, Gram-charlier densities, J Econ Dyn Control 25(10) (2001) 1457-1483.

[42] D. Barton, K. Dennis, The conditions under which Gram-Charlier and Edgeworth curves are positive definite and unimodal, Biometrika 39(3/4) (1952) 425-427.

[43] P. Kumar, M.S. Azam, S.K. Ghosh, Influence of stochastic roughness on performance of a Rayleigh step bearing operating under Thermo-elastohydrodynamic lubrication considering shear flow factor, Tribo Int 134 (2019) 264-280.

[44] U. Sudeep, N. Tandon and R.K. Pandey, Vibration studies of lubricated textured point contacts of bearing steels due to surface topographies: simulations and experiments, Tribol Int 102 (2016) 265-74.

[45] K. Maharshi, T. Mukhopadhyay, B. Roy, L. Roy, S. Dey, Stochastic dynamic behaviour of hydrodynamic journal bearings including the effect of surface roughness, Int J Mech Sci 142 (2018)370-383.

[46] T. Mukhopadhyay, S. Adhikari, Free vibration analysis of sandwich panels with randomly irregular honeycomb core, J Eng Mech 142 (2016) 06016008.

[47] J. Ma, H. Zhang, S. Lou, F. Chu, Z. Shi, F. Gu, D.A. Ball, Analytical and Experimental Investigation of Vibration Characteristics Induced by Tribofilm-Asperity Interactions in Hydrodynamic Journal Bearings, Mech Syst Signal Pr 150 (2021) 107227. 